\title{
US GEOLOGICAL SURVEY, DENVER, COLORADO RADIOCARBON DATES IV
}

\author{
I G YANG \\ US Geological Survey, Denver, Colorado 80225 \\ INTRODUCTION
}

This list contains the results of measurements of 167 groundwater samples made between September 1979 and December 1982. An additional 175 samples were measured, but these were submitted without detailed information, such as sampling depth, well location, etc; therefore, they were excluded from the following list.

${ }^{14} \mathrm{C}$ age calculations were based on the assumption that initial activity of the total dissolved inorganic carbon was $100 \%$ of modern ${ }^{14} \mathrm{C}$ activity. Although, this assumption generally is true for material of organic origin, it is not true for dissolved carbon in ground water. Estimation of initial activity of total dissolved carbon can be made from models. A review of the existing models and a new approach was made by Fontes and Garnier (1979). Interested readers may refer to these models for corrections of groundwater ages. ${ }^{14} \mathrm{C}$ half-life of $5568 \pm 30$ years was used in age calculations. Statistical errors quoted are $l \sigma$ counting errors, including sample, background, and standard error estimates. The age limit reported is calculated on the basis of $3 \sigma$ activity. The $\delta^{1:} \mathrm{C}$ values in table 1 were measured in an isotope laboratory of the US Geological Survey in Reston, Virginia, and calculated relative to Craig's Peedee Belemnite (PDB) limestone standard (Craig, 1957). Total alkalinity as bicarbonate values reported in table 1 was determined using techniques described by Brown, Skougstad, and Fishman (1970). Unless otherwise stated, all samples were collected and submitted by personnel of the US Geological Survey. Sample preparation and counting technique remain as previously reported (Yang \& Emerson, 1980; Yang, McAvoy, \& Emerson, 1981).

GROUNDWATER SAMPLES

Alabama

DE-69. Well Burkville A-2

$11,300 \pm 450$

Sample coll Jan 14, 1981, from Montgomery Co $\left(32^{\circ} 18^{\prime} 30^{\prime \prime} \mathrm{N}, 86^{\circ}\right.$ $31^{\prime} 25^{\prime \prime} \mathrm{W}$ ) from depth $225.55 \mathrm{~m}$. Alt of well head, $70.1 \mathrm{~m}$ above msl.

DE-70. Well A-5 W F Junkin

$6300 \pm 130$

Sample coll Jan 15, 1981, from Pickens Co $\left(33^{\circ} 26^{\prime} 49^{\prime \prime}\right.$ N, $87^{\circ} 56^{\prime} 29^{\prime \prime}$ W) from depth $67.67 \mathrm{~m}$. Hole drilled to $67.67 \mathrm{~m}$. Alt of well head, $109.42 \mathrm{~m}$ above msl.

DE-71. Well D-17 Bobby Bain

$2900 \pm 110$

Sample coll Jan 16, 1981, from Pickens Co $\left(33^{\circ} 28^{\prime} 47^{\prime \prime}\right.$ N, $88^{\circ} 12^{\prime} 37^{\prime \prime}$ W) from depth $48.77 \mathrm{~m}$. Hole drilled to $48.77 \mathrm{~m}$. Alt of well head, $88.39 \mathrm{~m}$ above msl. 
DE-72. Well 0-15 Hickman Farms

$18,900 \pm 1010$

Sample coll Jan 16, 1981, from Pickens Co $\left(33^{\circ} 16^{\prime} 37^{\prime \prime} \mathrm{N}, 88^{\circ} 15^{\prime} 44^{\prime \prime}\right.$

W) from depth $<60.96 \mathrm{~m}$. Hole drilled to $60.96 \mathrm{~m}$. Alt of well head, $82.3 \mathrm{~m}$ above msl.

DE-73. Well Burkville D-1

$21,900 \pm 1800$

Sample coll Apr 14, 1981, from Lowndes Co $\left(32^{\circ} 19^{\prime} 51^{\prime \prime} \mathrm{N}, 86^{\circ} 31^{\prime}\right.$ $41^{\prime \prime} \mathrm{W}$ ) from depth $335.28 \mathrm{~m}$. Hole drilled to $408.43 \mathrm{~m}$. Alt of well head, $46.94 \mathrm{~m}$ above $\mathrm{msl}$.

DE-74. Well Burkville B-2

$30,200 \pm 600$

Sample coll Apr 14, 1981, from Lowndes Co $\left(32^{\circ} 16^{\prime} 12^{\prime \prime} \mathrm{N}, 86^{\circ} 31^{\prime}\right.$ $13^{\prime \prime}$ W) from depth $158.5 \mathrm{~m}$. Hole drilled to $158.5 \mathrm{~m}$. Alt of well head, $65.53 \mathrm{~m}$ above $\mathrm{msl}$.

DE-75. Well CHO 1,Z 2 NR Melvin

$31,700 \pm 1330$

Sample coll Jan 27, 1981, from Choctaw Co $\left(31^{\circ} 55^{\prime} 53^{\prime \prime} \mathrm{N}, 88^{\circ} 27^{\prime}\right.$ $30^{\prime \prime}$ W) from depth $542.54 \mathrm{~m}$. Alt of well head, $106.68 \mathrm{~m}$ above msl.

DE-76. Well H 14A Jerome McArthur

$4800 \pm 680$

Sample coll July 28, 1982, from Russell Co $\left(32^{\circ} 19^{\prime} 45^{\prime \prime}\right.$ N, $85^{\circ} 10^{\prime} 05^{\prime \prime}$ W) from depth $25.91 \mathrm{~m}$. Hole drilled to $25.91 \mathrm{~m}$. Alt of well head, $140.21 \mathrm{~m}$ above msl.

DE-77. W ell L 12 City of Haneyville

$>40,000$

Sample coll July 8, 1982, from Lowndes Co $\left(32^{\circ} 10^{\prime} 58^{\prime \prime} \mathrm{N}, 86^{\circ} 34^{\prime}\right.$ $32^{\prime \prime}$ W) from depth $323.09 \mathrm{~m}$. Hole drilled to $323.39 \mathrm{~m}$. Alt of well head, $75.54 \mathrm{~m}$ above msl.

DE-78. Newtontown Well No. 2

$32,100 \pm 2120$

Sample coll July 13, 1982, from Dale Co $\left(31^{\circ} 19^{\prime} 21^{\prime \prime} \mathrm{N}, 85^{\circ} 35^{\prime} 33^{\prime \prime}\right.$ W) from depth $189.89 \mathrm{~m}$. Hole drilled to $219.46 \mathrm{~m}$. Alt of well head, $106.68 \mathrm{~m}$ above msl.

DE-79. Well P-2

$19,400 \pm 520$

Sample coll July 28, 1982, from Barbour Co $\left(31^{\circ} 53^{\prime} 08^{\prime \prime} \mathrm{N}, 85^{\circ} 35^{\prime}\right.$ $57^{\prime \prime} \mathrm{W}$ ) from depth $32 \mathrm{~m}$. Hole drilled to $55.17 \mathrm{~m}$. Alt of well head, $144.78 \mathrm{~m}$ above msl.

DE-80. Well Q Olympia Spa Golf Course $\quad 15,400 \pm 460$ Sample coll July 12, 1982 from Houston Co $\left(31^{\circ} 07^{\prime} 12^{\prime \prime} \mathrm{N}, 85^{\circ} 24^{\prime}\right.$ $24^{\prime \prime}$ W) from depth $85.34 \mathrm{~m}$. Hole drilled to $85.34 \mathrm{~m}$. Alt of well head, $82.3 \mathrm{~m}$ above misl.

DE-81. Well M Wyecott Plantation

$>\mathbf{3 6 , 8 0 0}$

Sample coll July 14 , 1982 , from Barbour Co $\left(31^{\circ} 58^{\prime} 14^{\prime \prime} \mathrm{N}, 85^{\circ} 19^{\prime}\right.$ $22^{\prime \prime}$ W) from depth $91.44 \mathrm{~m}$. Hole drilled to $91.44 \mathrm{~m}$. Alt of well head, $112.78 \mathrm{~m}$ above msl. 


\section{DE-82. S-1 City Well 3 at Clayton}

Sample coll July 14 , 1982, from Barbour Co $\left(31^{\circ} 52^{\prime} 08^{\prime \prime} \mathrm{N}, 85^{\circ} 24^{\prime}\right.$ $48^{\prime \prime}$ W) from depth $61.26 \mathrm{~m}$. Hole drilled to $59.44 \mathrm{~m}$. Alt of well head, $168.86 \mathrm{~m}$ above msl.

DE-83. Well A-1 Ben Bowden

$21,300 \pm 1340$

Sample coll July 15, 1982, from Barbour Co $\left(32^{\circ} 05^{\prime} 19^{\prime \prime} \mathrm{N}, 85^{\circ} 12^{\prime}\right.$ $54^{\prime \prime}$ W) from depth $160.68 \mathrm{~m}$. Hole drilled to $60.96 \mathrm{~m}$. Alt of well head, $71.32 \mathrm{~m}$ above $\mathrm{msl}$.

DE-84. Well B-3 Barbour County Board of Education $>\mathbf{3 0 , 7 0 0}$ Sample coll July 14, 1982, from Barbour Co $\left(32^{\circ} 04^{\prime} 31^{\prime \prime} \mathrm{N}, 85^{\circ} 19^{\prime}\right.$ $32^{\prime \prime}$ W) from depth $367.76 \mathrm{~m}$. Hole drilled to $367.76 \mathrm{~m}$. Alt of well head, $110.34 \mathrm{~m}$ above msl.

DE-85. Well B-11 Tom Smith

$4100 \pm 100$

Sample coll July 29, 1982, from Russell Co $\left(32^{\circ} 27^{\prime} 41^{\prime \prime}\right.$ N, $85^{\circ} 07^{\prime} 41^{\prime \prime}$ W) from depth $42.67 \mathrm{~m}$. Hole drilled to $42.67 \mathrm{~m}$.

DE-86. Well E-9 SH Robinson $10,900 \pm 180$

Sample coll July 13, 1982, from Henry Co $\left(31^{\circ} 37^{\prime} 38^{\prime \prime}\right.$ N, $85^{\circ} 16^{\prime} 30^{\prime \prime}$ W) from depth $103.94 \mathrm{~m}$.

DE-87. Well H-16 Lardonier-Crawford $18,700 \pm 660$

Sample coll July 27 , 1982, from Russell Co $\left(32^{\circ} 22^{\prime} 42^{\prime \prime} \mathrm{N}, 85^{\circ} 04^{\prime}\right.$ $48^{\prime \prime} \mathrm{W}$ ) from depth $114.91 \mathrm{~m}$. Hole drilled to $114.91 \mathrm{~m}$. Alt of well head, $80.77 \mathrm{~m}$ above $\mathrm{msl}$.

DE-88. Ozark City Well No. 2 $38,600 \pm 4150$

Sample coll July 13, 1982, from Dale Co (31 $27^{\prime} 23^{\prime \prime} \mathrm{N}, 85^{\circ} 38^{\prime} 42^{\prime \prime}$ W) from depth $213.36 \mathrm{~m}$.

DE-89. H-12 Greenville City Well

$>\mathbf{3 7 , 8 0 0}$

Sample coll July 8, 1982, from Butler Co $\left(31^{\circ} 49^{\prime} 47^{\prime \prime} \mathrm{N}, 86^{\circ} 36^{\prime} 40^{\prime \prime}\right.$ W) from depth $133.81 \mathrm{~m}$. Hole drilled to $175.87 \mathrm{~m}$. Alt of well head, $115.52 \mathrm{~m}$ above msl.

DE-90. Well P-I JB Neighbors

Sample coll July 7, 1982, from Macon Co $\left(32^{\circ} 28^{\prime} 33^{\prime \prime} \mathrm{N}, 86^{\circ} 41^{\prime} 03^{\prime \prime}\right.$ W) from depth $54.86 \mathrm{~m}$. Alt of well head, $56.69 \mathrm{~m}$ above msl.

DE-91. Well F-4 LB Bormeister $14,000 \pm 380$

Sample coll July 8, 1982, from Autauga Co $\left(32^{\circ} 36^{\prime} 48^{\prime \prime}\right.$ N, $86^{\circ} 40^{\prime} 16^{\prime \prime}$ W) from depth $36.58 \mathrm{~m}$. Hole drilled to $36.58 \mathrm{~m}$. Alt of well head, $89.92 \mathrm{~m}$ above msl.

\section{Colorado}

DE-92. Well TH 75-15A (C3-97-30 ACCI) $21,500 \pm 230$

Sample coll Oct 5, 1982, Rio Blanco Co $\left(39^{\circ} 45^{\prime} 40^{\prime \prime} \mathrm{N}, 108^{\circ} 19^{\prime} 12^{\prime \prime}\right.$ W) from depth $199.64 \mathrm{~m}$. 
US Geological Survey, Denver, Colorado Radiocarbon Dates IV 169

DE-93. Well TH-75-13B (C2-98-24 CBB3) >37,700

Sample coll Oct 6, 1982 from Rio Blanco Co $\left(39^{\circ} 51^{\prime} 36^{\prime \prime} \mathrm{N}, 108^{\circ} 21^{\prime}\right.$ $00^{\prime \prime}$ W) from depth $329.18 \mathrm{~m}$.

DE-94. Well TH 75-1A (B1-97-CAD1) 20,700 \pm 200

Sample coll Oct 13, 1982, from Rio Blanco Co $\left(40^{\circ} 02^{\prime} 18^{\prime \prime} \mathrm{N}, 108^{\circ} 17^{\prime}\right.$ $06^{\prime \prime}$ W) from depth $323.08 \mathrm{~m}$.

DE-95. Spring Yellow Creek No. I

$23,100 \pm 260$ (SC00109802aab)

Sample coll Oct 14, 1982, from Rio Blanco Co $\left(40^{\circ} 00^{\prime} 19^{\prime \prime} \mathrm{N}, 108^{\circ} 21^{\prime}\right.$ $10^{\prime \prime} \mathrm{W}$ ) from depth $213.36 \mathrm{~m}$.

\section{Florida}

DE-96. Well Fla For Srv Cmp Hndrsn

$>33,900$

Sample coll Feb 20, 1980, from Santa Rosa Co $\left(30^{\circ} 59^{\prime} 40^{\prime \prime} \mathrm{N}, 86^{\circ} 58^{\prime}\right.$ $06^{\prime \prime}$ W) from depth $248.41 \mathrm{~m}$. Alt of well head, $85.34 \mathrm{~m}$ above msl.

DE-97. USGS Test Well No. 1

$>37,600$

Sample coll Jan 23, 1980, from Santa Rosa Co (30 $32^{\prime} 41^{\prime \prime} \mathrm{N}, 86^{\circ} 54^{\prime}$ $04^{\prime \prime} \mathrm{W}$ ) from depth $457.2 \mathrm{~m}$. Alt of well head, $38.21 \mathrm{~m}$ above msl.

DE-98. USGS Test Well No. 2

$>41,600$

Sample coll Jan 8, 1980, from Santa Rosa Co $\left(30^{\circ} 42^{\prime} 52^{\prime \prime}\right.$ N, $87^{\circ} 00^{\prime}$ $22^{\prime \prime}$ W) from depth $393.19 \mathrm{~m}$. Alt of well head, $38.12 \mathrm{~m}$ above msl.

DE-99. Well North Monitor

$>36,300$

Sample coll Jan 22, 1980, from Escambia Co (30 $36^{\prime} 57^{\prime \prime} \mathrm{N}, 87^{\circ} 15^{\prime}$ $43^{\prime \prime}$ W) from depth $464.21 \mathrm{~m}$. Alt of well head, $2.39 \mathrm{~m}$ above msl.

DE-100. Well Deep Monitor North Am Cy >41,600

Sample coll Jan 7, 1980, from Santa Rosa Co $\left(30^{\circ} 35^{\prime} 14^{\prime \prime} \mathrm{N}, 87^{\circ} 05^{\prime}\right.$ $48^{\prime \prime}$ W) from clepth $509.02 \mathrm{~m}$. Alt of well head, $37.17 \mathrm{~m}$ above msl.

\section{DE-101. Well D-0349 J-0414}

$9800 \pm 150$

Sample coll June 19, 1981, from Duval Co $\left(30^{\circ} 24^{\prime} 16^{\prime \prime} \mathrm{N}, 81^{\circ} 52^{\prime} 26^{\prime \prime}\right.$

W) from depth $211.83 \mathrm{~m}$. Hole clrilled to $679.7 \mathrm{~m}$. Alt of well head, $24.38 \mathrm{~m}$ above msl.

DE-102. Well D-0349 J-0414

$6100 \pm 140$

Sample coll June 25, 1980, from Duval Co (30 $24^{\prime} 16^{\prime \prime} \mathrm{N}, 81^{\circ} 52^{\prime} 26^{\prime \prime}$

W) from depth $679.7 \mathrm{~m}$. Alt of well head, $24.38 \mathrm{~m}$ above $\mathrm{msl}$.

DE-103. Well 29S/38E-10

$>2000$

Sample coll July 28,1980 , from Brevard Co $\left(27^{\circ} 58^{\prime} 58^{\prime \prime} \mathrm{N}, 80^{\circ} 31^{\prime}\right.$ $18^{\prime \prime}$ W) from depth $100.28 \mathrm{~m}$. Alt of well head, $0.3 \mathrm{~m}$ above $\mathrm{msl}$.

DE-104. Well 30S/31E-I

Sample coll July 29, 1980, from Brevard Co $\left(27^{\circ} 52^{\prime} 08^{\prime \prime} \mathrm{N}, 80^{\circ} 27^{\prime}\right.$ $\left.\mathrm{I} 7^{\prime \prime} \mathrm{W}\right)$ from depth $74.68 \mathrm{~m}$. 
DE-105. Yates Well

Sample coll July 31 , 1980 , from Indian River Co $\left(27^{\circ} 48^{\prime} 57^{\prime \prime} \mathrm{N}, 80^{\circ}\right.$ $49^{\prime} 34^{\prime \prime}$ W). from depth $71.02 \mathrm{~m}$. Alt of well head, $8.53 \mathrm{~m}$ above msl.

DE-106. Well OS-273 NR Kenansville

$30,000 \pm 2000$

Sample coll July 31 , 1980 , from Hillsborough Co $\left(27^{\circ} 59^{\prime} 57^{\prime \prime} \mathrm{N}, 82^{\circ}\right.$ $52^{\prime} 31^{\prime \prime} \mathrm{W}$ ) from depth $83.52 \mathrm{~m}$. Hole drilled to $10.97 \mathrm{~m}$.

DE-107. Well 30S/37E-35

$28,800 \pm 2900$

Sample coll July 29, 1980 from Brevard Co $\left(27^{\circ} 49^{\prime} 25^{\prime \prime} \mathrm{N}, 80^{\circ} 36^{\prime}\right.$ $17^{\prime \prime}$ W) from depth $150.27 \mathrm{~m}$. Alt of well head, $7.73 \mathrm{~m}$ above $\mathrm{msl}$.

DE-108. Well 50S/35E-03g

$13,900 \pm 680$

Sample coll Oct 18 , 1980, from Broward Co $\left(26^{\circ} 10^{\prime} 16^{\prime \prime} \mathrm{N}, 80^{\circ} 49^{\prime}\right.$ $26^{\prime \prime}$ W) from depth $284.68 \mathrm{~m}$. Hole drilled to $856.79 \mathrm{~m}$. Alt of well head, $4.71 \mathrm{~m}$ above $\mathrm{msl}$.

DE-109. Well 50S/35E-03g

$>24,500$

Sample coll Mar 9, 1981, from Broward Co $\left(26^{\circ} 10^{\prime} 16^{\prime \prime} \mathrm{N}, 80^{\circ} 49^{\prime}\right.$ $26^{\prime \prime}$ W) from depth $308.46 \mathrm{~m}$.

DE-110. Well 50S/35E-03g

$22,900 \pm 1060$

Sample coll Mar 8, 1981, from Broward Co $\left(26^{\circ} 10^{\prime} 16^{\prime \prime} \mathrm{N}, 80^{\circ} 49^{\prime} 26^{\prime \prime}\right.$ W) from depth $326.9 \mathrm{~m}$.

DE-111. Well 50S/35E-03g

$24,500 \pm 1150$

Sample coll Mar 7, 1981, from Broward Co $\left(26^{\circ} 10^{\prime} 16^{\prime \prime} \mathrm{N}, 80^{\circ} 49^{\prime} 2^{\prime \prime}\right.$ W) from depth $466.34 \mathrm{~m}$.

DE-112. Well 50S/35E-03g

$9600 \pm 480$

Sample coll Mar 3, 1981, from Broward Co $\left(26^{\circ} 10^{\prime} 16^{\prime \prime}\right.$ N, $80^{\circ} 49^{\prime} 26^{\prime \prime}$ W) from depth $746.76 \mathrm{~m}$.

DE-113. Tide Water Well 1 $6300 \pm 130$

Sample coll Sept 7, 1981, from Marion Co $\left(29^{\circ} 07^{\prime} 43^{\prime \prime} \mathrm{N}, 82^{\circ} 34^{\prime} 15^{\prime \prime}\right.$ W) from depth $238.96 \mathrm{~m}$. Alt of well head, $22.51 \mathrm{~m}$ above $\mathrm{msl}$.

\section{DE-114. Well 50S/35E-03g}

$>\mathbf{3 7 , 8 0 0}$

Sample coll Oct 19, 1981, from Broward Co $\left(26^{\circ} 10^{\prime} 16^{\prime \prime} \mathrm{N}, 80^{\circ} 49^{\prime}\right.$ $26^{\prime \prime}$ W) from depth $248.41 \mathrm{~m}$. Fole drilled to $856.79 \mathrm{~m}$. Alt of well head, $4.71 \mathrm{~m}$ above $\mathrm{msl}$.

\section{DE-115. Well 50S/35E-03g}

$>\mathbf{2 0 , 7 0 0}$

Sample coll Oct 19, 1981 , from Broward Co $\left(26^{\circ} 10^{\prime} 16^{\prime \prime} \mathrm{N}, 80^{\circ} 49^{\prime}\right.$ $26^{\prime \prime}$ W) from depth $762 \mathrm{~m}$. Hole drilled to $856.79 \mathrm{~m}$. Alt of well head, $4.7 \mathrm{~lm}$ above msl.

DE-116. Well 56S/40E-21

$7100 \pm 190$

Sample coll Oct 22,1981 , from Hendry Co $\left(26^{\circ} 32^{\prime} 55^{\prime \prime} \mathrm{N}, 80^{\circ} 19^{\prime}\right.$ $57^{\prime \prime}$ W) from depth $819.61 \mathrm{~m}$. Hole drilled to $825.09 \mathrm{~m}$. Alt of well head, $3.05 \mathrm{~m}$ above $\mathrm{msl}$. 
US Geological Survey, Denver, Colorado Radiocarbon Dates IV 171

DE-117. Well 50S/42E-14bi

$>\mathbf{2 6 , 9 0 0}$

Sample coll Oct 21, 1981, from Broward Co $\left(26^{\circ} 05^{\prime} 55^{\prime \prime} \mathrm{N}, 80^{\circ} 00^{\prime}\right.$ $73^{\prime \prime}$ W) from depth $782.73 \mathrm{~m}$.

DE-118. Well 50S/35E-03g

$17,100 \pm 700$

Sample coll Oct 19, 1981, from Broward Co $\left(26^{\circ} 10^{\prime} 16^{\prime \prime} \mathrm{N}, 80^{\circ} 49^{\prime}\right.$ $26^{\prime \prime} \mathrm{W}$ ) from depth $274.32 \mathrm{~m}$. Hole drilled to $856.79 \mathrm{~m}$. Alt of well head, $4.71 \mathrm{~m}$ above $\mathrm{msl}$.

DE-119. Well 50S/42E-14bi $27,900 \pm 1000$

Sample coll Oct 21, 1981, from Broward Co $\left(26^{\circ} 05^{\prime} 55^{\prime \prime} \mathrm{N}, 80^{\circ} 00^{\prime}\right.$ 73 " W) from depth $311.2 \mathrm{~m}$.

DE-120. Well 56S/40E-21 21,600 \pm 260

Sample coll Oct 22, 1981, from Palm Beach Co $\left(26^{\circ} 32^{\prime} 55^{\prime \prime} \mathrm{N}, 80^{\circ}\right.$ $19^{\prime} 57^{\prime \prime} \mathrm{W}$ ) from depth $307.85 \mathrm{~m}$. Hole drilled to $825.09 \mathrm{~m}$. Alt of well head, $3.05 \mathrm{~m}$ above $\mathrm{msl}$.

DE-121. Firetower Well nr Codys Corner $\quad 8300 \pm 140$

Sample coll Feb 17, 1982, from Flagler Co $\left(29^{\circ} 18^{\prime} 18^{\prime \prime} \mathrm{N}, 81^{\circ} 19^{\prime} 05^{\prime \prime}\right.$ W) from depth $42.67 \mathrm{~m}$. Alt of well head, $7.62 \mathrm{~m}$ above msl.

DE-122. 12S/29E-33 Harper Well

$>\mathbf{2 6 , 8 0 0}$

Sample coll Feb 18, 1982, from Flagler Co $\left(29^{\circ} 25^{\prime} 21^{\prime \prime} \mathrm{N}, 81^{\circ} 22^{\prime} 23^{\prime \prime}\right.$ W) from depth $91.44 \mathrm{~m}$. Alt of well head, $2.13 \mathrm{~m}$ above msl.

DE-123. Newbold Irrigation Well at Crescent City $10,600 \pm 340$ Sample coll Feb 16, 1982, from Putnam Co $\left(29^{\circ} 27^{\prime} 41^{\prime \prime}\right.$ N, $81^{\circ} 30^{\prime} 58^{\prime \prime}$ W) from depth $24.38 \mathrm{~m}$. Alt of well head, $1.52 \mathrm{~m}$ above msl.

DE-124. Well Yarborough Home $7100 \pm 150$

Sample coll Mar 3, 1982, from Seminole Co $\left(28^{\circ} 44^{\prime} 11^{\prime \prime}\right.$ N, $81^{\circ} 26^{\prime} 58^{\prime \prime}$ W) from depth $45.72 \mathrm{~m}$. Alt of well head, $10.73 \mathrm{~m}$ above msl.

DE-125. Well 13S/30E-06 27,800 \pm 2190

Sample coll Feb 18, 1982, from Flagler Co $\left(29^{\circ} 23^{\prime} 42^{\prime \prime}\right.$ N, $81^{\circ} 18^{\prime} 37^{\prime \prime}$ W) from depth $86.86 \mathrm{~m}$. Alt of well head, $4.32 \mathrm{~m}$ above msl.

DE-126. Well Seminole Pkwy at Sesame Blvd $13,300 \pm 260$ Sample coll Feb 22, 1982, from Flagler Co $\left(29^{\circ} 25^{\prime} 59^{\prime \prime} \mathrm{N}, 81^{\circ} 11^{\prime} 11^{\prime \prime}\right.$ W) from depth $89.92 \mathrm{~m}$. Alt of well head, $8.53 \mathrm{~m}$ above msl.

DE-127. Stock Well at St. Johns Park

$>\mathbf{2 2 , 3 0 0}$

Sample coll Feb 22, 1982, from Flagler Co $\left(29^{\circ} 25^{\prime} 28^{\prime \prime} \mathrm{N}, 81^{\circ} 25^{\prime} 53^{\prime \prime}\right.$ W) from depth $91.44 \mathrm{~m}$. Alt of well head, $2.13 \mathrm{~m}$ above msl.

DE-128. Palm Coast Well SW-92

$11,200 \pm 130$

Sample coll Mar 1, 1982, from Flagler Co $\left(29^{\circ} 36^{\prime} 36^{\prime \prime} \mathrm{N}, 81^{\circ} 18^{\prime} 09^{\prime \prime}\right.$ W) from depth $26.82 \mathrm{~m}$. Alt of well head, $9.14 \mathrm{~m}$ above msl. 
DE-129. Palm Coast Well SW-91

Sample coll Mar 1, 1982, from Flagler Co $\left(29^{\circ} 34^{\prime} 06^{\prime \prime} \mathrm{N}, 81^{\circ} 17^{\prime} 46^{\prime \prime}\right.$

W) from depth $24.99 \mathrm{~m}$. Alt of well head, $9.73 \mathrm{~m}$ above msl.

DE-130. Well USGS TW 1

\section{Georgia}

Sample coll June 9, 1981, from Ware Co (31 $07^{\prime} 06^{\prime \prime} \mathrm{N}, 82^{\circ} 15^{\prime} 51^{\prime \prime}$ W) from depth $565.71 \mathrm{~m}$. Alt of well head, $45.7 \mathrm{~m}$ above $\mathrm{msl}$.

DE-131. Well J P Stevens 4

$8800 \pm 140$

Sample coll Aug 22, 1981, from Jefferson Co $\left(33^{\circ} 00^{\prime} 14^{\prime \prime} \mathrm{N}, 82^{\circ} 27^{\prime}\right.$ $39^{\prime \prime}$ W) from depth $129.24 \mathrm{~m}$. Alt of well head, $85.34 \mathrm{~m}$ above msl.

DE-132. Well Wrens, GA;3

$2000 \pm 130$

Sample coll Sept 3, 1981, from Jefferson Co $\left(33^{\circ} 11^{\prime} 57^{\prime \prime}\right.$ N, 82 $2^{\circ} 3^{\prime}$ $25^{\prime \prime}$ W) from depth $42.98 \mathrm{~m}$. Alt of well head, 128.93 above msl.

DE-133. Well Thiele Co, Reedy CR 1

$1500 \pm 270$

Sample coll Sept 4, 1981, from Jefferson Co $\left(33^{\circ} 10^{\prime} 49^{\prime \prime} \mathrm{N}, 82^{\circ} 27^{\prime}\right.$ $1 \mathrm{I}^{\prime \prime}$ W) from depth $45.79 \mathrm{~m}$. Alt of well head, $138.44 \mathrm{~m}$ above msl.

DE-134. Well J M Huber Co., 2

$3700 \pm 240$

Sample coll Sept 3, 1981, from Jefferson Co $\left(33^{\circ} 16^{\prime} 52^{\prime \prime} \mathrm{N}, 82^{\circ} 24^{\prime}\right.$ $34^{\prime \prime}$ W) from depth $95.1 \mathrm{~m}$. Alt of well head, $131.06 \mathrm{~m}$ above msl.

DE-135. Well Gibson, GA 3

Sample coll Sept 4, 1981, from Glascock Co $\left(33^{\circ} 13^{\prime} 59^{\prime \prime} \mathrm{N}, 82^{\circ} 35^{\prime}\right.$ $56^{\prime \prime} \mathrm{W}$ ) from depth $61.87 \mathrm{~m}$. Alt of well head, $132.56 \mathrm{~m}$ above msl.

DE-136. Well King Finishing No. 1

$32,900 \pm 2700$

Sample coll Aug 19, 1981, from Screven Co $\left(32^{\circ} 36^{\prime} 14^{\prime \prime} \mathrm{N}, 81^{\circ} 44^{\prime}\right.$ $21^{\prime \prime}$ W) from depth $404.17 \mathrm{~m}$. Alt of well head, $48.77 \mathrm{~m}$ above msl.

DE-137. Chalker Well

Sample coll Aug 21, 1981, from Warren Co $\left(33^{\circ} 20^{\prime} 00^{\prime \prime} \mathrm{N}, 82^{\circ} 38^{\prime}\right.$ $08^{\prime \prime} \mathrm{W}$ ) from depth $6.09 \mathrm{~m}$.

DE-138. Well Pincushion TW-3

$19,700 \pm 370$

Sample coll Jan 28, 1982, from Laurens Co $\left(32^{\circ} 30^{\prime} 30^{\prime \prime} \mathrm{N}, 83^{\circ} 03^{\prime}\right.$ $00^{\prime \prime}$ W) from depth $377.92 \mathrm{~m}$. Hole drilled to $513.59 \mathrm{~m}$. Alt of well head, $85.95 \mathrm{~m}$ above $\mathrm{msl}$.

DE-139. Well Ellaville GA 5

$8300 \pm 290$

Sample coll Aug 3, 1982, from Schley Co $\left(32^{\circ} 14^{\prime} 08^{\prime \prime} \mathrm{N}, 84^{\circ} 18^{\prime} 20^{\prime \prime}\right.$ W) from depth $195.07 \mathrm{~m}$. Hole drilled to $198.12 \mathrm{~m}$. Alt of well head, $173.13 \mathrm{~m}$ above msl.

DE-140. Well Breeland, Lee

$600 \pm 570$

Sample coll Aug 5, 1982, from Schley Co $\left(32^{\circ} 17^{\prime} 47^{\prime \prime} \mathrm{N}, 84^{\circ} 17^{\prime} 24^{\prime \prime}\right.$ W) from depth $39.01 \mathrm{~m}$. Hole drilled to $39.01 \mathrm{~m}$. Alt of well head, $146.91 \mathrm{~m}$ above msl. 


\section{DE-141. Well Albany TW-10}

$27,900 \pm 840$

Sample coll July 20, 1982, from Dougherty Co $\left(31^{\circ} 35^{\prime} 30^{\prime \prime} \mathrm{N}, 84^{\circ} 10^{\prime}\right.$ $30^{\prime \prime}$ W) from depth $256.03 \mathrm{~m}$.

DE-142. Crouch Well H-5

$1150 \pm 70$

Sample coll July 28 , 1982, from Chattahoochee Co $\left(32^{\circ} 23^{\prime} 22^{\prime \prime} \mathrm{N}, 85^{\circ}\right.$ $07^{\prime} 13^{\prime \prime}$ W) from depth $32 \mathrm{~m}$.

DE-143. Well Kolomoki State Park No. $2 \quad 10,200 \pm 300$

Sample coll July 20, 1982, from Early Co $\left(31^{\circ} 28^{\prime} 05^{\prime \prime} \mathrm{N}, 84^{\circ} 55^{\prime} 40^{\prime \prime}\right.$ W) from depth $44.2 \mathrm{~m}$.

DE-144. Well Albany TW-1

$>40,000$

Sample coll July 19, 1982, from Dougherty Co $\left(31^{\circ} 31^{\prime} 05^{\prime \prime} \mathrm{N}, 84^{\circ} 06^{\prime}\right.$ $43^{\prime \prime}$ W) from depth $449.28 \mathrm{~m}$.

DE-145. Well Fort Gains, GA-3

$>\mathbf{3 6 , 5 0 0}$

Sample coll July 21, 1982, from Glay Co $\left(31^{\circ} 36^{\prime} 38^{\prime \prime} \mathrm{N}, 85^{\circ} 03^{\prime} 21^{\prime \prime}\right.$ W) from depth $109.73 \mathrm{~m}$. Hole drilled to $112.78 \mathrm{~m}$. Alt of well head, $45.42 \mathrm{~m}$ above msl.

DE-146. Well Wainwright-House

$1300 \pm 210$

Sample coll July 22, 1982, from Taylor Co $\left(32^{\circ} 40^{\prime} 12^{\prime \prime} \mathrm{N}, 84^{\circ} 11^{\prime} 04^{\prime \prime}\right.$ W) from depth $21.34 \mathrm{~m}$. Hole drilled to $21.34 \mathrm{~m}$. Alt of well head, $165.51 \mathrm{~m}$ above msl.

DE-147. Well Georgetown No. 1

$>\mathbf{3 8 , 4 0 0}$

Sample coll July 21, 1982, from Quitman Co $\left(31^{\circ} 53^{\prime} 10^{\prime \prime} \mathrm{N}, 85^{\circ} 05^{\prime}\right.$ $57^{\prime \prime}$ W) from depth $416.05 \mathrm{~m}$.

DE-148. Well Georgetown No. 2

$\mathbf{2 4 , 3 0 0} \pm \mathbf{7 5 0}$

Sample coll July 21, 1982, from Quitman Co $\left(31^{\circ} 52^{\prime} 21^{\prime \prime} \mathrm{N}, 85^{\circ} 04^{\prime}\right.$ $5]^{\prime \prime}$ W) from depth $536.45 \mathrm{~m}$.

DE-149. Omaha School Well

$15,900 \pm 180$

Sample coll Aug 4, 1982, from Stewart Co $\left(32^{\circ} 08^{\prime} 32^{\prime \prime} \mathrm{N}, 85^{\circ} 00^{\prime} 04^{\prime \prime}\right.$ W) from depth $96.32 \mathrm{~m}$.

DE-150. Well Omaha, GA-1

$>\mathbf{3 1 , 0 0 0}$

Sample coll Aug 4, 1982, from Stewart Co $\left(32^{\circ} 08^{\prime} 59^{\prime \prime}\right.$ N, $85^{\circ} 00^{\prime} 37^{\prime \prime}$ $W$ ) from depth $295.05 \mathrm{~m}$. Hole drilled to $312.42 \mathrm{~m}$. Alt of well head, $91.74 \mathrm{~m}$ above msl.

DE-151. Well BIBB Plant Laurel 2 $2000 \pm 160$

Sample coll July 22, 1982, from Taylor Co $\left(32^{\circ} 30^{\prime} 58^{\prime \prime} \mathrm{N}, 84^{\circ} 07^{\prime} 19^{\prime \prime}\right.$ W) from depth $58.83 \mathrm{~m}$. Hole drilled to $58.83 \mathrm{~m}$. Alt of well head, $129.54 \mathrm{~m}$ above msl.

DE-152. Well Singletary Bancroft

$25,800 \pm 960$

Sample coll July 20, 1982, from Early Co $\left(31^{\circ} 24^{\prime} 45^{\prime \prime} \mathrm{N}, 84^{\circ} 49^{\prime} 41^{\prime \prime}\right.$ W) from depth $234.7 \mathrm{~m}$. Alt of well head, $233.78 \mathrm{~m}$ above msl. 
DE-153. Well Wainwright 2

Sample coll July 22, 1982, from Taylor Co $\left(32^{\circ} 33^{\prime} 04^{\prime \prime} \mathrm{N}, 84^{\circ} 10^{\prime} 11^{\prime \prime}\right.$ W) from depth $185.01 \mathrm{~m}$. Hole drilled to $185.01 \mathrm{~m}$. Alt of well head, $192.63 \mathrm{~m}$ above $\mathrm{msl}$.

DE-154. Well Weston GA (Old 1)

$\mathbf{1 0 , 5 0 0} \pm \mathbf{1 7 0}$

Sample coll Aug 4, 1982, from Webster Co (31 $58^{\prime} 41^{\prime \prime}$ N, 84 $36^{\prime} 55^{\prime \prime}$

W) from depth $81.69 \mathrm{~m}$. Hole drilled to $81.69 \mathrm{~m}$. Alt of well head, $159.11 \mathrm{~m}$ above msl.

\section{Idaho}

DE-155. Well 04S/01E-34bad1

$21,500 \pm 600$

Sample coll Jan 27, 1982, from Owyhee Co $\left(43^{\circ} 02^{\prime} 36^{\prime \prime} \mathrm{N}, 116^{\circ} 19^{\prime}\right.$ $26^{\prime \prime}$ W) from depth $408.30 \mathrm{~m}$. Alt of well head, $783.34 \mathrm{~m}$ above msl.

DE-156. Well 05S/03E-26bcbl

$24,600 \pm 1850$

Sample coll Jan 26,1982 from Owyhee Co $\left(42^{\circ} 57^{\prime} 50^{\prime \prime} \mathrm{N}, 116^{\circ} 04^{\prime}\right.$ $32^{\prime \prime}$ W) from depth $905.26 \mathrm{~m}$. Alt of well head, $757.43 \mathrm{~m}$ above msl.

DE-157. Well 08S/14E-30acd2

$25,800 \pm 1570$

Sample coll Jan 18, 1982, from Jerome Co $\left(42^{\circ} 42^{\prime} 14^{\prime \prime} \mathrm{N}, 114^{\circ} 51^{\prime}\right.$ $21^{\prime \prime} \mathrm{W}$ ) from depth $128.02 \mathrm{~m}$. Alt of well head, $882.09 \mathrm{~m}$ above msl.

Iowa

DE-158. Well Webster 6

$>37,400$

Sample coll Feb 24, 1982, from Hamilton Cio $\left(42^{\circ} 28^{\prime} 55^{\prime \prime} \mathrm{N}, 93^{\circ} 48^{\prime}\right.$ $15^{\prime \prime}$ W) from depth $609.6 \mathrm{~m}$.

DE-159. Ode Bolt 2 Town Well

$33,800 \pm 1700$

Sample coll Feb 23, 1982, from Carroll Co $\left(42^{\circ} 18^{\prime} 31^{\prime \prime}\right.$ N, $95^{\circ} 15^{\prime} 21^{\prime \prime}$ W) from depth $650.75 \mathrm{~m}$.

DE-160. Well 07-227W-10edca

$29,400 \pm 930$

Sample coll Feb 23, 1982, from Monroe Co $\left(41^{\circ} 02^{\prime} 35^{\prime \prime}\right.$ N, $93^{\circ} 56^{\prime} 49^{\prime \prime}$

W) from depth $949.15 \mathrm{~m}$. Alt of well head, $371.86 \mathrm{~m}$ above msl.

DE-161. Well 08-007W -25bdb

$24,600 \pm 510$

Sample coll Feb 24, 1982, from Linn Co $\left(41^{\circ} 42^{\prime} 35^{\prime \prime} \mathrm{N}, 91^{\circ} 36^{\prime} 41^{\prime \prime}\right.$ W) from depth $524.25 \mathrm{~m}$. Alt of well head, $247.5 \mathrm{~m}$ above msl.

DE-162. Well 08-307W-01badc

$26,400 \pm 920$

Sample coll Feb 24, 1982, from Monroe Co $\left(42^{\circ} 02^{\prime} 00^{\prime \prime} \mathrm{N}, 91^{\circ} 36^{\prime} 30^{\prime \prime}\right.$ W) from depth $477.0 \mathrm{Im}$. Alt of well head, $243.84 \mathrm{~m}$ above msl.

DE-163. Well 07-623W-3Idadd

Sample coll Feb 22, 1982, from Warren Co $\left(41^{\circ} 20^{\prime} 25^{\prime \prime}\right.$ N, $93^{\circ} 32^{\prime} 22^{\prime \prime}$ W) from depth $742.18 \mathrm{~m}$. Alt of well head, $258.78 \mathrm{~m}$ above msl. 


\section{Mississippi}

DE-164. Well C040 H Miller

$19,700 \pm 1110$

Sample coll Jan 19, 1981, from Noxubee Co $\left(33^{\circ} 13^{\prime} 08^{\prime \prime} \mathrm{N}, 88^{\circ} 36^{\prime}\right.$ $51^{\prime \prime}$ W) from depth $493.78 \mathrm{~m}$. Alt of well head, $78.64 \mathrm{~m}$ above msl.

DE-165. Well B013 Hoffman

$22,100 \pm 300$

Sample coll Jan 10, 1981, from Noxubee Co $\left(33^{\circ} 15^{\prime} 28^{\prime \prime} \mathrm{N}, 88^{\circ} 37^{\prime}\right.$ $07^{\prime \prime} \mathrm{W}$ ) from depth $231.65 \mathrm{~m}$. Alt of well head, $88.39 \mathrm{~m}$ above msl.

DE-166. Brooksville Town Well

$21,600 \pm 450$

Sample coll Jan 12, 1981, from Noxubee Co $\left(33^{\circ} 13^{\prime} 57^{\prime \prime} \mathrm{N}, 88^{\circ} 34^{\prime}\right.$ $52^{\prime \prime}$ W) from depth $287.12 \mathrm{~m}$.

DE-167. Well D018 Holcut Cairo WA

$4200 \pm 110$

Sample coll Jan 9, 1981, from Jackson Co (34 $44^{\prime} 55^{\prime \prime} \mathrm{N}, 88^{\circ} 21^{\prime} 40^{\prime \prime}$ W) from depth $92.96 \mathrm{~m}$. Alt of well head, $179.83 \mathrm{~m}$ above msl.

DE-168. Well Hol0 Ashland

$14,400 \pm 420$

Sample coll Jan 7, 1981, from Amite Co $\left(34^{\circ} 51^{\prime} 00^{\prime \prime} \mathrm{N}, 89^{\circ} 10^{\prime} 48^{\prime \prime}\right.$ W) from depth $280.42 \mathrm{~m}$. Alt of well head, $186.84 \mathrm{~m}$ above msl.

\section{DE-169. Well B011 Walnut}

$\mathbf{2 8 , 8 0 0} \pm \mathbf{2 9 0 0}$

Sample coll Jan 6, 1981, from Alcorn Co (34 $57^{\prime} 24^{\prime \prime} \mathrm{N}, 88^{\circ} 54^{\prime} 00^{\prime \prime}$ W) from depth $292.91 \mathrm{~m}$. Alt of well head, $161.54 \mathrm{~m}$ above $\mathrm{msl}$.

DE-170. Well C018 Black Belt Exp Sta

$7700 \pm 900$

Sample coll Jan 12, 1981, from Noxubee Co $\left(33^{\circ} 15^{\prime} 31^{\prime \prime} \mathrm{N}, 88^{\circ} 33^{\prime}\right.$ $41^{\prime \prime}$ W) from depth $393.19 \mathrm{~m}$. Alt of well head, $85.95 \mathrm{~m}$ above msl.

\section{DE-171. Well BO07 Walnut}

$10,900 \pm 170$

Sample coll Jan 8, 1981, from 'Tippah Co (34 $56^{\prime} 57^{\prime \prime} \mathrm{N}, 88^{\circ} 53^{\prime} 49^{\prime \prime}$ W) from deptl $44.81 \mathrm{~m}$. Alt of well head, $143.26 \mathrm{~m}$ above msl.

\section{DE-172. Well B002 Abrams}

$13,300 \pm 200$

Sample coll Jan 10, 1981, from Noxubee Co $\left(33^{\circ} 11^{\prime} 45^{\prime \prime} \mathrm{N}, 88^{\circ} 37^{\prime}\right.$ $04^{\prime \prime}$ W) from depth $264.57 \mathrm{~m}$. Alt of wcll head, $85.65 \mathrm{~m}$ above msl.

\section{DE-173. Well E003 Mathis}

$1200 \pm 370$

Sample coll Jan 8, 1981, from Alcorn Cio (34 $56^{\prime} 00^{\prime \prime} \mathrm{N}, 88^{\circ} 48^{\prime} 26^{\prime \prime}$ W) from depth $38.1 \mathrm{~m}$. Alt of well head, $155.45 \mathrm{~m}$ above msl.

DE-174. Well L006 Mashulaville $33,500 \pm 1550$

Sample coll Jan 11, 1981, from Noxubee Co $\left(33^{\circ} 05^{\prime} 28^{\prime \prime} \mathrm{N}, 88^{\circ} 44^{\prime}\right.$ $30^{\prime \prime} \mathrm{W}$ ) from depth $557.78 \mathrm{~m}$. Alt of well head, $80.77 \mathrm{~m}$ above msl.

DE-175. Well D005 Byhalia

$29,800 \pm 900$

Sample coll Jan 7, 1981, from Harrison Co (34 $52^{\prime} 20^{\prime \prime}$ N, $89^{\circ} 46^{\prime} 23^{\prime \prime}$ W) from depth $499.87 \mathrm{~m}$. Alt of well head, $109.73 \mathrm{~m}$ above msl. 
DE-176. Well A010 Columbus AFB No. $2 \quad 16,200 \pm 500$

Sample coll Jan 9, 1981, from Lowndes Co $\left(33^{\circ} 37^{\prime} 32^{\prime \prime}\right.$ N, $88^{\circ} 26^{\prime} 36^{\prime \prime}$ W) from depth $134.72 \mathrm{~m}$. Alt of well head, $61.87 \mathrm{~m}$ above $\mathrm{msl}$.

DE-177. Well C020 Butler $\quad 31,300 \pm 900$

Sample coll Jan 11, 1981, from Noxubee Co $\left(33^{\circ} 13^{\prime} 57^{\prime \prime} \mathrm{N}, 88^{\circ} 34^{\prime}\right.$ $08^{\prime \prime}$ W) from depth $215.8 \mathrm{~m}$. Alt of well head, $79.25 \mathrm{~m}$ above msl.

\section{Nevada}

\section{DE-178. Well 210/S13/E63-23dd $\quad 20,700 \pm 230$}

Sample coll Dec 23, 1980, from Clark Co $\left(36^{\circ} 47^{\prime} 44^{\prime \prime} \mathrm{N}, 114^{\circ} 53^{\prime} 32^{\prime \prime}\right.$ W) from depth $203.91 \mathrm{~m}$. Alt of well head, $664.46 \mathrm{~m}$ above msl.

\section{DE-179. Well 179/N12/E63-12bdabl $\quad 9600 \pm 110$}

Sample coll Jan 19, 1981, from White Pine Co $\left(38^{\circ} 55^{\prime} 16^{\prime \prime} \mathrm{N}, 114^{\circ}\right.$ $50^{\prime} 21^{\prime \prime} \mathrm{W}$ ) from depth $288.95 \mathrm{~m}$.

\section{DE-180. Well 210/S13/E63-23dd $\quad 20,800 \pm 240$}

Sample coll July 22, 1981 , from Clark Co $\left(36^{\circ} 47^{\prime} 44^{\prime \prime}\right.$ N, $114^{\circ} 53^{\prime} 32^{\prime \prime}$ W) from depth $203.91 \mathrm{~m}$. Alt of well head, $664.46 \mathrm{~m}$ above msl.

DE-181. Well VE 29A-2-H

$3800 \pm 120$

Sample coll Jan 8, 1982, from Nye Co (36 $\left.56^{\prime} 29^{\prime \prime} \mathrm{N}, 116^{\circ} 22^{\prime} 26^{\prime \prime} \mathrm{W}\right)$ from depth $335.7 \mathrm{~m}$.

DE-182. Well VE 29A-2-H $\quad 4100 \pm 120$

Sample coll Jan 15, 1982, from Nye Co $\left(36^{\circ} 56^{\prime} 29^{\prime \prime} \mathrm{N}, 116^{\circ} 22^{\prime} 26^{\prime \prime}\right.$ W) from depth $150.12 \mathrm{~m}$.

$\begin{array}{ll}\text { DE-183. USW-H } 6 & \mathbf{1 4 , 6 0 0} \pm 100\end{array}$

Sample coll Oct 16,1982 , from Nye Co $\left(36^{\circ} 50^{\prime} 49^{\prime \prime} \mathrm{N}, 116^{\circ} 28^{\prime} 55^{\prime \prime}\right.$ W) from depth $557.75 \mathrm{~m}$.

New Mexico

\section{DE-184. Oscura Range Camp Well \\ $15,700 \pm 330$}

Sample coll July 29 , 1982, from Lincoln Co $\left(33^{\circ} 29^{\prime} 54^{\prime \prime} \mathrm{N}, 106^{\circ} 10^{\prime}\right.$ 22 " W) from depth $41.76 \mathrm{~m}$.

DE-185. Carolina Welding Well

$300 \pm 140$

Sample coll Aug 12, 1981, from Scotland Co (34 $44^{\prime} 18^{\prime \prime} \mathrm{N}, 79^{\circ} 27^{\prime}$ 13 "W) from depth $7.62 \mathrm{~m}$.

\section{DE-186. Rowland, RB-39}

$9000 \pm 220$

Sample coll Aug 12, 1981, from Robeson Co $\left(34^{\circ} 32^{\prime} 21^{\prime \prime} \mathrm{N}, 79^{\circ} 17^{\prime}\right.$ $02 "$ W) from depth $128.32 \mathrm{~m}$.

\section{DE-187. Rowland, RB-41}

$6500 \pm 250$

Sample coll Aug 12, 1981 from Robeson Co $\left(34^{\circ} 32^{\prime} 38^{\prime \prime} \mathrm{N}, 79^{\circ} 17^{\prime}\right.$ $43^{\prime \prime}$ W) from depth $76.2 \mathrm{~m}$. 
US Geological Survey, Denver, Colorado Radiocarbon Dates IV 177

DE-188. Laurinburg, SC-18 $2700 \pm \mathbf{2 8 0}$

Sample coll Aug 12, 1981, from Scotland Co $\left(34^{\circ} 47^{\prime} 02^{\prime \prime}\right.$ N, $79^{\circ} 26^{\prime}$ $30^{\prime \prime}$ W) from depth $42.67 \mathrm{~m}$.

DE-189. Wood

$$
\text { Oregon }
$$

Wood sample coll Oct 1979, from Douglas Co $\left(43^{\circ} 27^{\prime} 16^{\prime \prime} \mathrm{N}, 124^{\circ}\right.$ $16^{\prime} 24^{\prime \prime} \mathrm{W}$ ) from depth $49.07 \mathrm{~m}$.

DE-190. Shell

$7500 \pm 190$

Shell sample coll Oct 1979, from Douglas Co $\left(43^{\circ} 27^{\prime} 16^{\prime \prime} \mathrm{N}, 124^{\circ} 16^{\prime}\right.$ $24^{\prime \prime}$ W) from depth $33.53 \mathrm{~m}$.

\section{South Carolina}

DE-191. Well BFT-457

$\mathbf{2 8 , 6 0 0} \pm \mathbf{5 2 0}$

Sample coll Sept 10, 1981, from Beaufort Co $\left(32^{\circ} 19^{\prime} 39^{\prime \prime} \mathrm{N}, 80^{\circ} 27^{\prime}\right.$ $42^{\prime \prime}$ W) from depth $832.1 \mathrm{~m}$. Hole drilled to $953.11 \mathrm{~m}$. Alt of well head, $2.13 \mathrm{~m}$ above $\mathrm{msl}$.

DE-192. Well Isle of Palms $\quad \mathbf{2 8 , 4 0 0} \pm \mathbf{5 1 0}$

Sample coll Aug 7, 1981, from Charleston Co $\left(32^{\circ} 47^{\prime} 15^{\prime \prime} \mathrm{N}, 79^{\circ} 49^{\prime}\right.$ $17^{\prime \prime}$ W) from depth $609.6 \mathrm{~m}$.

DE-193. Well BFT-454

$30,100 \pm 620$

Sample coll Sept 10, 1981, from Beaufort Co $\left(32^{\circ} 14^{\prime} 46^{\prime \prime} \mathrm{N}, 80^{\circ} 44^{\prime}\right.$ $40^{\prime \prime}$ W) from depth $923.54 \mathrm{~m}$. Hole drilled to $924.76 \mathrm{~m}$. Alt of well head, $2.05 \mathrm{~m}$ above $\mathrm{msl}$.

\section{DE-194. Well MRN-42 Marion TWN No. $3 \quad 10,600 \pm 150$}

Sample coll Aug 13, 1981, from Marion Co (34 $10^{\prime} 26^{\prime \prime}$ N, $79^{\circ} 23^{\prime} 47^{\prime \prime}$ W) from depth $173.74 \mathrm{~m}$. Alt of well head, $21.34 \mathrm{~m}$ above msl.

\section{DE-195. Well MRN-38 Marion TWN No. $2 \quad 28,600 \pm 1290$}

Sample coll Aug 13, 1981, from Marion Co (34 $11^{\prime} 01^{\prime \prime}$ N, $79^{\circ} 23^{\prime} 47^{\prime \prime}$ W) from depth $60.96 \mathrm{~m}$. Hole drilled to $60.96 \mathrm{~m}$. Alt of well head, $21.95 \mathrm{~m}$ above msl.

DE-196. Well Brookgreen No. 1

$5100 \pm 120$

Sample coll Aug 5, 1981, from Horry Co (33 $31^{\prime} 13^{\prime \prime} \mathrm{N}, 79^{\circ} 05^{\prime} 35^{\prime \prime}$ W) from depth $33.52 \mathrm{~m}$.

DE-197. Well Brookgreen No. 2

Sample coll Aug 5, 1981, from Horry Co $\left(33^{\circ} 30^{\prime} 59^{\prime \prime} \mathrm{N}, 79^{\circ} 05^{\prime} 32^{\prime \prime}\right.$ W) from depth $205.74 \mathrm{~m}$.

DE-198. Well H0-299

Sample coll Aug 5, 1981, from Horry Co $\left(33^{\circ} 53^{\prime} 33^{\prime \prime} \mathrm{N}, 78^{\circ} 35^{\prime} 20^{\prime \prime}\right.$ W) from depth $381 \mathrm{~m}$. 
DE-199. Well CHN-64-CHN

$33,100 \pm 930$

Sample coll Aug 7, 1981, from Charleston Co $\left(32^{\circ} 47^{\prime} 08^{\prime \prime} \mathrm{N}, 79^{\circ} 55^{\prime}\right.$ $55^{\prime \prime}$ W) from depth $408.43 \mathrm{~m}$. Hole drilled to $406.91 \mathrm{~m}$. Alt of well head, $4.88 \mathrm{~m}$ above $\mathrm{msl}$.

DE-200. Well Conway W-2

$32,600 \pm 870$

Sample coll Aug 6, 1981, from Horry Co (33 $51^{\prime} 01^{\prime \prime} \mathrm{N}, 79^{\circ} 04^{\prime} 08^{\prime \prime}$ W) from depth $204.06 \mathrm{~m}$.

\section{DE-201. Well BFT-11}

$36,200 \pm 2920$

Sample coll Sept 11, 1981, from Beaufort Co $\left(32^{\circ} 21^{\prime} 10^{\prime \prime} \mathrm{N}, 80^{\circ} 41^{\prime}\right.$ $22^{\prime \prime}$ W) from depth $850.39 \mathrm{~m}$. Hole drilled $849.17 \mathrm{~m}$. Alt of well head, $3.66 \mathrm{~m}$ above $\mathrm{msl}$.

DE-202. Well BFT-10

$\mathbf{3 2 , 8 0 0} \pm \mathbf{8 9 0}$

Sample coll Sept 11, 1981, from Beaufort Co $\left(32^{\circ} 19^{\prime} 46^{\prime \prime} \mathrm{N}, 80^{\circ} 42^{\prime}\right.$ $26^{\prime \prime} \mathrm{W}$ ) from depth $905.26 \mathrm{~m}$. Hole drilled to $1045.46 \mathrm{~m}$. Alt of well head, $3.66 \mathrm{~m}$ above msl.

DE-203. Well GEO-88

$>41,800$

Sample coll Aug 25, 1981, from Georgetown Co $\left(33^{\circ} 15^{\prime} 08^{\prime \prime} \mathrm{N}, 79^{\circ}\right.$ $16^{\prime} 24^{\prime \prime}$ W) from depth $563.88 \mathrm{~m}$. Hole drilled to $394.72 \mathrm{~m}$. Alt of well head, $1.83 \mathrm{~m}$ above $\mathrm{msl}$.

DE-204. Hodge Well

$31,300 \pm 800$

Sample coll Aug 24, 1981, from Charleston Co $\left(32^{\circ} 55^{\prime} 35^{\prime \prime} \mathrm{N}, 79^{\circ} 44^{\prime}\right.$ $16^{\prime \prime}$ W) from depth $129.54 \mathrm{~m}$.

DE-205. Well BRK-9

$\mathbf{2 6 , 8 0 0} \pm \mathbf{4 7 0}$

Sample coll Aug 24, 1981, from Charleston Co $\left(33^{\circ} 11^{\prime} 50^{\prime \prime} \mathrm{N}, 80^{\circ} 00^{\prime}\right.$ $40^{\prime \prime} \mathrm{W}$ ) from depth $45.72 \mathrm{~m}$. Hole drilled to $45.72 \mathrm{~m}$. Alt of well head, $15.24 \mathrm{~m}$ above $\mathrm{msl}$.

DE-206. Well N Myrtle Beach-3

$6700 \pm 130$

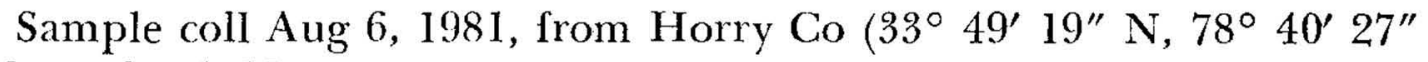
W) from depth $32 \mathrm{~m}$.

DE-207. MRN-78, Test Well No. 2 $24,500 \pm 340$

Sample coll Apr 1, 1982, from Horry Co $\left(33^{\circ} 51^{\prime} 43^{\prime \prime} \mathrm{N}, 79^{\circ} 19^{\prime} 50^{\prime \prime}\right.$ W) from depth $347.47 \mathrm{~m}$.

DE-208. MRN-78, Test Well No. 2 $22,600 \pm 250$

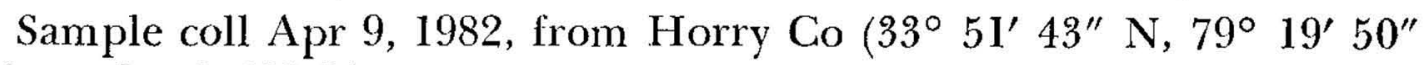
W) from depth $313.94 \mathrm{~m}$.

DE-209. MRN-78, Test Well No. 2 $29,900 \pm 580$

Sample coll Apr 19, 1982, from Horry Co (33 $51^{\prime} 43^{\prime \prime}$ N, $79^{\circ} 19^{\prime} 50^{\prime \prime}$ W) from depth $253.29 \mathrm{~m}$. 
US Geological Survey, Denver, Colorado Radiocarbon Dates IV 179

DE-210. MRN-78, Test Well No. 2

$29,600 \pm 970$

Sample coll Apr 24, 1982, from Horry Co (33 $51^{\prime} 43^{\prime \prime} \mathrm{N}, 79^{\circ} 19^{\prime} 50^{\prime \prime}$ W) from depth $234.09 \mathrm{~m}$.

DE-211. MRN-78, Test Well No. 2

$\mathbf{2 8 , 4 0 0} \pm \mathbf{5 0 0}$

Sample coll Apr 30, 1982, from Horry Co (33 $51^{\prime} 43^{\prime \prime} \mathrm{N}, 79^{\circ} 19^{\prime} 50^{\prime \prime}$ W) from depth $163.68 \mathrm{~m}$.

DE-212. MRN-78, Test Well No. 2

$>\mathbf{3 8 , 1 0 0}$

Sample coll May 2, 1982, from Horry Co ( $33^{\circ} 5 \mathrm{I}^{\prime} 43^{\prime \prime} \mathrm{N}, 79^{\circ} 19^{\prime} 50^{\prime \prime}$ W) from depth $108.2 \mathrm{~m}$.

\section{South Dakota}

DE-213. Well 93N/55W-04bbc

$\mathbf{2 8 , 3 0 0} \pm \mathbf{5 8 0}$

Sample coll May 7, 1980, from Yankton Co $\left(42^{\circ} 54^{\prime} 40^{\prime \prime} \mathrm{N}, 97^{\circ} 21^{\prime} 25^{\prime \prime}\right.$

W) from depth $117.65 \mathrm{~m}$. Hole drilled to $124.05 \mathrm{~m}$. Alt of well head, $365.76 \mathrm{~m}$ above msl.

DE-214. Well 96N/63W-68cda

$33,200 \pm 1570$

Sample coll May 8, 1980, from Charles Mix Co $\left(43^{\circ} 08^{\prime} 26^{\prime \prime} \mathrm{N}, 98^{\circ} 19^{\prime}\right.$ $06^{\prime \prime}$ W) from depth $201.63 \mathrm{~m}$. Hole drilled to $204.83 \mathrm{~m}$. Alt of well head, $433.73 \mathrm{~m}$ above msl.

DE-215. Well 91N/49W-19bcea

$22,000 \pm 320$

Sample coll May 6, 1980, from Union Co $\left(42^{\circ} 41^{\prime} 13^{\prime \prime} \mathrm{N}, 96^{\circ} 41^{\prime} 13^{\prime \prime}\right.$ W) from depth $99.06 \mathrm{~m}$. Hole drilled to $99.06 \mathrm{~m}$.

DE-216. Well 116N/70W-07ada

$31,600 \pm 780$

Sample coll May 13, 1980, from Hand Co $\left(44^{\circ} 52^{\prime} 36^{\prime \prime}\right.$ N, $99^{\circ} 17^{\prime} 38^{\prime \prime}$

W) from depth $581.66 \mathrm{~m}$. Hole drilled to $587.96 \mathrm{~m}$. Alt of well head, $582.17 \mathrm{~m}$ above $\mathrm{msl}$.

DE-217. Well 109N/70W-04babd

$33,700 \pm 1140$

Sample coll May 14, 1980, from Hand Co $\left(44^{\circ} 16^{\prime} 53^{\prime \prime} \mathrm{N}, 99^{\circ} 15^{\prime} 33^{\prime \prime}\right.$

W) from depth $573.94 \mathrm{~m}$. Hole drilled to $592.84 \mathrm{~m}$. Alt of well head, $570.59 \mathrm{~m}$ above $\mathrm{msl}$.

DE-218. Well 113N/66W-25dada2

$29,400 \pm 1110$

Sample coll May 12, 1980, from Hand Co $\left(44^{\circ} 34^{\prime} 01^{\prime \prime}\right.$ N, $98^{\circ} 42^{\prime} 26^{\prime \prime}$ W) from depth $352.04 \mathrm{~m}$.

DE-219. Well 107N/65W-5bbba

$\mathbf{2 3 , 5 0 0} \pm \mathbf{5 4 0}$

Sample coll May 15, 1980, from Jerauld Co $\left(44^{\circ} 06^{\prime} 34^{\prime \prime} \mathrm{N}, 98^{\circ} 40^{\prime} 03^{\prime \prime}\right.$ W) from depth $429.16 \mathrm{~m}$. Hole drilled to $432.82 \mathrm{~m}$. Alt of well head, $576.07 \mathrm{~m}$ above $\mathrm{msl}$.

DE-220. Well 104N/61W-36ddaa $30,268 \pm 793$

Sample coll May 19, 1980, from Davison Co $\left(43^{\circ} 45^{\prime} 55^{\prime \prime} \mathrm{N}, 98^{\circ} 05^{\prime}\right.$ $12^{\prime \prime}$ W) from depth $103.63 \mathrm{~m}$. Hole drilled to $103.63 \mathrm{~m}$. Alt of well head, $394.72 \mathrm{~m}$ above msl. 
DE-221. Well 105N/58W-31bace $26,400 \pm 520$

Sample coll May 20, 1980, from Miner Co $\left(43^{\circ} 51^{\prime} 42^{\prime \prime} \mathrm{N}, 97^{\circ} 50^{\prime} 39^{\prime \prime}\right.$ W) from depth $192.02 \mathrm{~m}$. Hole drilled to $192.02 \mathrm{~m}$. Alt of well head, $398.68 \mathrm{~m}$ above msl.

DE-222. Well 128N/66W-08abbc $\quad 32,000 \pm 1330$

Sample coll May 22, 1980, from McPherson Co $\left(45^{\circ} 55^{\prime} 27^{\prime \prime}\right.$ N, $98^{\circ} 49^{\prime}$ 03" W) from depth $547.73 \mathrm{~m}$. Hole drilled to $581.25 \mathrm{~m}$.

DE-223. Well 2N/8E-17edd $\quad 5000 \pm 130$

Sample coll June 19, 1980, from Pennington Co $\left(44^{\circ} 07^{\prime} 35^{\prime \prime} \mathrm{N}, 103^{\circ}\right.$ $10^{\prime} 56^{\prime \prime} \mathrm{W}$ ) from depth $528.82 \mathrm{~m}$. Hole drilled to $528.82 \mathrm{~m}$.

DE-224. Well 106N/76W-3b

$>\mathbf{2 5 , 3 0 0}$

Sample coll June 26, 1980, from Buffalo Co $\left(44^{\circ} 00^{\prime} 50^{\prime \prime} \mathrm{N}, 99^{\circ} 56^{\prime}\right.$ $06^{\prime \prime}$ W) from depth $431.6 \mathrm{~m}$. Hole drilled to $437.99 \mathrm{~m}$.

DE-225. Well 9N/26E-12add $>\mathbf{3 5 , 3 0 0}$

Sample coll June 30 , 1980, from Potter Co $\left(44^{\circ} 45^{\prime} 16^{\prime \prime} \mathrm{N}, 100^{\circ} 53^{\prime}\right.$ $37^{\prime \prime}$ W) from depth $514.05 \mathrm{~m}$. Hole drilled to $548.6 \mathrm{~m}$.

DE-226. Well 02S/31E-35cace

$>42,200$

Sample coll July 1, 1980, from Jones Co $\left(43^{\circ} 49^{\prime} 31^{\prime \prime} \mathrm{N}, 100^{\circ} 22^{\prime} 53^{\prime \prime}\right.$ W) from depth $503.38 \mathrm{~m}$. Hole drilled to $539.5 \mathrm{~m}$.

DE-227. Well 112N/74W-4dc

$>42,200$

Sample coll July 2, 1980, from Hughes Co (44 $31^{\prime} 42^{\prime \prime}$ N, $99^{\circ} 43^{\prime} 54^{\prime \prime}$ W) from depth $429.01 \mathrm{~m}$. Hole drilled to $445.01 \mathrm{~m}$.

DE-228. Well SH:Q-40

Tennessee

Sample coll Mar 31, 1982, from Shelby Co (35 $11^{\prime} 09^{\prime \prime}$ N, $89^{\circ} 51^{\prime} 29^{\prime \prime}$ W) from depth $134.42 \mathrm{~m}$.

DE-229. Well SH:0-231 Mallory ML6N $3500 \pm 60$

Sample coll Mar 31, 1982, from Shelby Co (35 $09^{\prime} 17^{\prime \prime}$ N, $90^{\circ} 01^{\prime} 20^{\prime \prime}$ W) from depth $157.89 \mathrm{~m}$. Hole drilled to $157.89 \mathrm{~m}$.

DE-230. Well SH:T-16 Shelby Forest Campground $\mathbf{7 3 0 0} \pm \mathbf{7 0}$

Sample coll June 3, 1982, from Shelby Co $\left(35^{\circ} 20^{\prime} 45^{\prime \prime}\right.$ N, $90^{\circ} 02^{\prime} 49^{\prime \prime}$ W) from depth $178 \mathrm{~m}$. Hole drilled to $178 \mathrm{~m}$.

DE-231. Well DOE No. 6

\section{Utah}

Sample coll Jan 8, 1980, from Grand Co $\left(38^{\circ} 50^{\prime} 33^{\prime \prime} \mathrm{N}, 109^{\circ} 44^{\prime} 21^{\prime \prime}\right.$ W) from depth $148.13 \mathrm{~m}$.

DE-232. Well DOE No. 4 $41,400 \pm 2700$

Sample coll Jan 31, 1980, from Grand Co $\left(38^{\circ} 50^{\prime} 24^{\prime \prime} \mathrm{N}, 109^{\circ} 44^{\prime} 08^{\prime \prime}\right.$ W) from depth $149.68 \mathrm{~m}$. 
DE-233. W ell DOE No. 8

$16,700 \pm 200$

Sample coll Feb 20, 1980, from Grand Co $\left(38^{\circ} 50^{\prime} 34^{\prime \prime} \mathrm{N}, 109^{\circ} 44^{\prime} 18^{\prime \prime}\right.$ W) from depth $159.72 \mathrm{~m}$.

DE-234. Well DOE No. 9

$25,600 \pm 700$

Sample coll Feb 26, 1980, from Grand Co $\left(38^{\circ} 50^{\prime} 34^{\prime \prime}\right.$ N, $109^{\circ} 44^{\prime} 18^{\prime \prime}$ W) from depth $136.25 \mathrm{~m}$.

DE-235. Well DOE No. 5

$32,000 \pm 800$

Sample coll Mar 12, 1980, from Grand Co ( $38^{\circ} 50^{\prime} 31^{\prime \prime}$ N, $109^{\circ} 44^{\prime} 05^{\prime \prime}$

W) from depth $125.27 \mathrm{~m}$.

\section{REFFRFNCES}

Brown, Eugene, Skougstad, M W, and Fishman, M J, 1970, US Geological Survey techniques of water resources investigations: Book 5, Chap A1, p 42-44.

Craig, Harmon, 1957, Isotopic standards for carbon and oxygen and factors for massspectrometric analysis of carbon dioxide: Geochim et Cosmochim Acta, v 12, p 133149.

Fontes, J C and Garnier, J M, 1979, Determination of the initial ${ }^{14} \mathrm{C}$ activity of the total dissolved carbon. A review of the existing models and a new approach: Water Resources Research, v 15, no. 2, p 399-413.

Schroder, L J, Beetem, W A, Claassen, H C, and Emerson, R L, 1973, US Geological Survey, Denver, Colorado radiocarbon dates I: Radiocarbon, v 15, p 469-478.

Schroder, L J, Emerson, R L, and Beetem, W A, 1978, US Geological Survey, Denver, Colorado radiocarbon dates II: Radiocarbon, v 20, p 200-209.

Yang, I C and Emerson, R L, 1980, Teflon vials for low-level ${ }^{14} \mathrm{C}$ liquid scintillation counting, in Peng, C T, Horrocks, D L, and Alpen, E L, eds, Internatl conf on liquid scintillation counting, recent applications and developments: New York, Academic Press, v 2, p 181-197.

Yang, I C, McAvoy, R L, and Emerson, R L, 1981, US Geological Survey, Denver, Colorado radiocarbon dates III: Radiocarbon, v 23, p 24-32. 
TABLE, 1

Summary of $\delta^{13} \mathrm{C}$ and alkalinity for water sources

\begin{tabular}{|c|c|c|c|c|}
\hline $\begin{array}{c}\text { Sample } \\
\text { no. }\end{array}$ & $\begin{array}{l}\text { Colln } \\
\text { date }\end{array}$ & $\begin{array}{c}\delta^{13} \mathrm{C} \\
(\%, \mathrm{PDB})\end{array}$ & $\begin{array}{c}\text { Total alkalinity } \\
\text { as bicarbonate } \\
(\mathrm{mg} / \mathrm{L})\end{array}$ & $\begin{array}{l}\text { Water source } \\
\text { by state }\end{array}$ \\
\hline DE-69 & $1 / 14 / 81$ & -16.7 & 45 & Alabama \\
\hline-70 & $1 / 15 / 81$ & -18.6 & 46 & $"$ \\
\hline-71 & $1 / 16 / 81$ & -18.9 & 66 & " \\
\hline .72 & $1 / 16 / 81$ & -19.1 & 94 & ,. \\
\hline-73 & $4 / 14 / 81$ & -19.1 & 48 & $"$ \\
\hline-74 & $4 / 14 / 81$ & -10.3 & 630 & " \\
\hline-75 & $1 / 27 / 81$ & -12.6 & 450 & $"$ \\
\hline-76 & $7 / 28 / 82$ & -21.0 & 5 & $"$ \\
\hline-77 & $7 / 8 / 82$ & -15.6 & 130 & $"$ \\
\hline .78 & $7 / 13 / 82$ & -9.5 & 180 & $"$ \\
\hline .79 & $7 / 28 / 82$ & -13.2 & 160 & $"$ \\
\hline-80 & $7 / 12 / 82$ & -14.6 & 130 & $"$ \\
\hline-81 & $7 / 14 / 82$ & -10.7 & 200 & ,. \\
\hline .82 & $7 / 14 / 82$ & -20.0 & 36 & ", \\
\hline .83 & $7 / 15 / 82$ & -8.5 & 52 & $"$ \\
\hline-84 & $7 / 14 / 82$ & -17.8 & 78 & $"$ \\
\hline-85 & $7 / 29 / 82$ & -22.5 & 40 & $"$ \\
\hline-86 & $7 / 19 / 82$ & -11.8 & 230 & " \\
\hline-87 & $7 / 27 / 82$ & -19.3 & 69 & ", \\
\hline-88 & $7 / 13 / 82$ & -6.5 & - & " \\
\hline-89 & $7 / 8 / 82$ & -8.8 & $\overline{250}$ & $"$ \\
\hline-90 & $7 / 7 / 82$ & -23.2 & 17 & $"$ \\
\hline-91 & $7 / 8 / 82$ & -24.2 & 16 & $"$ \\
\hline-92 & $10 / 5 / 82$ & -4.6 & - & Colorado \\
\hline-93 & $10 / 6 / 82$ & -9.5 & - & " \\
\hline-94 & $10 / 13 / 82$ & -8.7 & - & $"$ \\
\hline .95 & $10 / 14 / 82$ & -8.2 & — & $"$ \\
\hline-96 & $2 / 20 / 80$ & -3.8 & 170 & Florida \\
\hline-97 & $1 / 23 / 80$ & -3.5 & 391 & " \\
\hline-98 & $1 / 8 / 80$ & -2.9 & 378 & ", \\
\hline-99 & $1 / 22 / 80$ & -4.9 & 320 & $"$ \\
\hline-100 & $1 / 7 / 80$ & -3.2 & 435 & $"$ \\
\hline-101 & $6 / 19 / 80$ & -9.8 & - & $"$ \\
\hline-102 & $6 / 25 / 80$ & -6.6 & - & $"$ \\
\hline-103 & $7 / 28 / 80$ & -5.7 & 170 & " \\
\hline-104 & $7 / 29 / 80$ & -4.4 & 200 & $"$ \\
\hline-105 & $7 / 31 / 80$ & -7.7 & 150 & $"$ \\
\hline-106 & $7 / 31 / 80$ & -7.4 & 180 & ". \\
\hline-107 & $7 / 29 / 80$ & -7.4 & 170 & " \\
\hline-108 & $10 / 18 / 80$ & -3.1 & 97 & $"$ \\
\hline-109 & $3 / 9 / 81$ & -2.4 & - & $"$ \\
\hline-110 & $3 / 8 / 81$ & -2.6 & - & $"$ \\
\hline-111 & $3 / 7 / 81$ & -1.6 & $\ldots$ & ," \\
\hline-112 & $3 / 3 / 81$ & -3.8 & - & $"$ \\
\hline-113 & $9 / 7 / 81$ & -9.2 & $\ldots$ & $"$ \\
\hline-114 & $10 / 19 / 81$ & -1.8 & - & ", \\
\hline-115 & $10 / 19 / 81$ & -7.3 & - &, \\
\hline-116 & $10 / 22 / 82$ & -5.3 & $\ldots$ & $"$ \\
\hline-117 & $10 / 21 / 81$ & - & $\ldots$ & " \\
\hline-118 & $10 / 19 / 81$ & -1.2 & - & $"$ \\
\hline-119 & $10 / 21 / 81$ & - & -- & $"$ \\
\hline-120 & $10 / 22 / 81$ & -3.9 & - & $"$ \\
\hline-121 & $2 / 17 / 82$ & -11.1 & - & $"$ \\
\hline-122 & $2 / 18 / 82$ & -9.0 & - & $"$ \\
\hline-123 & $2 / 16 / 82$ & -6.9 & - & ", \\
\hline-124 & $3 / 3 / 82$ & -9.3 & - & ", \\
\hline-125 & $2 / 18 / 82$ & -8.8 & - & $"$ \\
\hline
\end{tabular}


US Geological Survey, Denver, Colorado Radiocarbon Dates IV 183

TABLE 1 (continued)

\begin{tabular}{|c|c|c|c|c|}
\hline $\begin{array}{l}\text { Sample } \\
\text { no. }\end{array}$ & $\begin{array}{l}\text { Colln } \\
\text { date }\end{array}$ & $\begin{array}{c}\delta^{13} \mathrm{C} \\
(\% o \mathrm{PDB})\end{array}$ & $\begin{array}{c}\text { Total alkalinity } \\
\text { as bicarbonate } \\
(\mathrm{mg} / \mathrm{L})\end{array}$ & $\begin{array}{c}\text { Water source } \\
\text { by state }\end{array}$ \\
\hline $\mathrm{DE}-126$ & $2 / 22 / 82$ & -7.3 & - & Florida \\
\hline-127 & $2 / 22 / 82$ & -9.0 & - & $"$ \\
\hline-128 & $3 / 1 / 82$ & -11.5 & - & $"$ \\
\hline-129 & $3 / 1 / 82$ & -10.6 & 一 & $"$ \\
\hline-130 & $6 / 9 / 81$ & -12.1 & $\overrightarrow{140}$ & Georgia \\
\hline-131 & $8 / 22 / 81$ & -19.1 & 46 & $"{ }^{2}$ \\
\hline-132 & $9 / 3 / 81$ & -22.9 & 7 & $"$ \\
\hline-133 & $9 / 4 / 81$ & -19.1 & 28 & $"$ \\
\hline-134 & $9 / 3 / 81$ & -25.7 & 2 & ", \\
\hline-135 & $9 / 4 / 81$ & -25.4 & 1 & $"$ \\
\hline-136 & $8 / 19 / 81$ & -16.4 & 110 & $"$ \\
\hline-137 & $8 / 21 / 81$ & -21.8 & - & $"$ \\
\hline-138 & $1 / 28 / 82$ & -20.6 & 46 & $"$ \\
\hline-139 & $8 / 3 / 82$ & -22.3 & 25 & " \\
\hline-140 & $8 / 5 / 82$ & -20.4 & 2 & $"$ \\
\hline .141 & $7 / 20 / 82$ & -4.8 & - & $"$ \\
\hline-142 & $7 / 28 / 82$ & -21.4 & - & $"$ \\
\hline-143 & $7 / 20 / 82$ & -12.2 & - & $"$ \\
\hline-144 & $7 / 19 / 82$ & -2.0 & - & , \\
\hline-145 & $7 / 21 / 82$ & -4.2 & 210 & $"$ \\
\hline-146 & $7 / 22 / 82$ & -22.4 & 1 & $"$ \\
\hline-147 & $7 / 21 / 82$ & -10.4 & $\ldots$ & , \\
\hline-148 & $7 / 21 / 82$ & -17.2 & - & $"$ \\
\hline-149 & $8 / 4 / 82$ & -13.4 & - & " \\
\hline-150 & $8 / 4 / 82$ & -16.2 & 88 & , \\
\hline-151 & $7 / 22 / 82$ & -23.2 & 2 & $"$ \\
\hline-152 & $7 / 20 / 82$ & -6.3 & 160 & $"$ \\
\hline-153 & $7 / 22 / 82$ & -20.9 & 2 & $"$ \\
\hline-154 & $8 / 4 / 82$ & -9.1 & 180 & $"$ \\
\hline-155 & $1 / 27 / 82$ & -11.5 & - & Idaho \\
\hline-156 & $1 / 26 / 82$ & -14.0 & - & $"$ \\
\hline-157 & $1 / 18 / 82$ & -7.0 & - & $"$ \\
\hline-158 & $2 / 24 / 82$ & -- & - & Iowa \\
\hline-159 & $2 / 23 / 82$ & - & - & $"$ \\
\hline-160 & $2 / 23 / 82$ & - & - & $"$ \\
\hline-161 & $2 / 24 / 82$ & - & - & ", \\
\hline-162 & $2 / 24 / 82$ & - & - & ", \\
\hline-163 & $2 / 22 / 82$ & - & - & $"$ \\
\hline-164 & $1 / 19 / 81$ & -16.3 & 90 & Mississippi \\
\hline-165 & $1 / 10 / 81$ & -5.2 & 400 & "1 \\
\hline-166 & $1 / 12 / 81$ & -17.4 & - & $"$ \\
\hline-167 & $1 / 9 / 81$ & -18.7 & 71 & ". \\
\hline-168 & $1 / 7 / 81$ & -13.2 & 190 & " \\
\hline-169 & $1 / 6 / 81$ & -16.3 & 150 & " \\
\hline-170 & $1 / 12 / 81$ & -19.6 & 80 & " \\
\hline-171 & $1 / 8 / 81$ & -12.4 & 210 & " \\
\hline-172 & $1 / 10 / 81$ & -9.6 & 180 & $"$ \\
\hline-173 & $1 / 8 / 81$ & -19.3 & 6 & $"$ \\
\hline-174 & $1 / 11 / 81$ & -15.9 & 200 & $"$ \\
\hline-175 & $1 / 7 / 81$ & -12.1 & 480 & $"$ \\
\hline-176 & $1 / 9 / 81$ & -18.3 & 71 & ", \\
\hline-177 & $1 / 11 / 81$ & -8.2 & 470 & $"$ \\
\hline-178 & $12 / 23 / 80$ & -10.6 & - & Nevada \\
\hline-179 & $1 / 19 / 81$ & -14.6 & - & " \\
\hline-180 & $7 / 22 / 81$ & -14.4 & - & $"$ \\
\hline-181 & $1 / 8 / 82$ & - & - & ", \\
\hline-182 & $1 / 15 / 82$ & - & - & $"$ \\
\hline-183 & $10 / 16 / 82$ & -7.5 & -- & $"$ \\
\hline-184 & $7 / 29 / 82$ & -3.5 & -- & New Mexico \\
\hline
\end{tabular}


184

I C Yang

Tarle 1 (continued)

\begin{tabular}{|c|c|c|c|c|}
\hline $\begin{array}{l}\text { Sample } \\
\text { no. }\end{array}$ & $\begin{array}{l}\text { Colln } \\
\text { date }\end{array}$ & 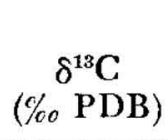 & $\begin{array}{c}\text { Total alkalinity } \\
\text { as bicarbonate } \\
(\mathrm{mg} / \mathrm{L})\end{array}$ & $\begin{array}{c}\text { Water source } \\
\text { by state }\end{array}$ \\
\hline DE-185 & $8 / 12 / 8 \mathrm{I}$ & -21.5 & - & North Carolina \\
\hline-186 & $8 / 12 / 81$ & -18.3 & 48 & " \\
\hline-187 & $8 / 12 / 81$ & -13.4 & 69 & $"$ \\
\hline-188 & $8 / 12 / 81$ & -22.7 & - & $"$ \\
\hline-189 & $10 / 79$ & - & - & Oregon \\
\hline-190 & $10 / 79$ & - & - & 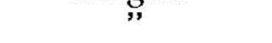 \\
\hline-191 & $9 / 10 / 81$ & -6.8 & 1430 & South Garolina \\
\hline-192 & $8 / 7 / 81$ & -6.6 & 1050 & $"$ \\
\hline-193 & $9 / 10 / 81$ & -6.8 & 1100 & $"$ \\
\hline-194 & $8 / 13 / 81$ & -19.3 & 92 & $"$ \\
\hline-195 & $8 / 13 / 81$ & $-\mathrm{I} 6.8$ & 110 & $"$ \\
\hline-196 & $8 / 5 / 81$ & -11.1 & 190 & $"$ \\
\hline-197 & $8 / 5 / 81$ & -5.2 & 600 & $"$ \\
\hline-198 & $8 / 5 / 8 \mathrm{I}$ & -9.0 & 460 & $"$ \\
\hline-199 & $8 / 7 / 81$ & -6.6 & 860 & $"$ \\
\hline-200 & $8 / 6 / 81$ & -8.9 & - & $"$ \\
\hline-201 & $9 / 11 / 81$ & -5.6 & 1080 & $"$ \\
\hline-202 & $9 / 11 / 81$ & -6.0 & 1250 & $"$ \\
\hline-203 & $8 / 25 / 81$ & -6.8 & 1240 & $"$ \\
\hline-204 & $8 / 24 / 81$ & -4.6 & - & $"$ \\
\hline-205 & $8 / 24 / 81$ & -7.7 & 260 & ", \\
\hline-206 & $8 / 6 / 81$ & -9.6 & 590 & $"$ \\
\hline-207 & $4 / 1 / 82$ & -13.6 & - & ,. \\
\hline-208 & $4 / 9 / 82$ & -12.9 & - & ," \\
\hline-209 & $4 / 19 / 82$ & -12.2 & - & ". \\
\hline-210 & $4 / 24 / 82$ & -10.3 & - & $"$ \\
\hline-211 & $4 / 30 / 82$ & - & - & , \\
\hline-212 & $5 / 2 / 82$ & - & - & $"$ \\
\hline-213 & $5 / 7 / 80$ & -6.1 & - & South Dakota \\
\hline-214 & $5 / 8 / 80$ & -9.1 & - & $"$ \\
\hline-215 & $5 / 6 / 80$ & -7.5 & - & $"$ \\
\hline-216 & $5 / 13 / 80$ & -3.9 & - & ", \\
\hline-217 & $5 / 14 / 80$ & -3.4 & - & " \\
\hline-218 & $5 / 12 / 80$ & -5.0 & - & ", \\
\hline-219 & $5 / 15 / 80$ & -5.1 & - & ." \\
\hline-220 & $5 / 19 / 80$ & -4.6 & - & $"$ \\
\hline-221 & $5 / 20 / 80$ & -5.5 & - & $"$ \\
\hline-222 & $5 / 22 / 80$ & -4.8 & - & ", \\
\hline-223 & $6 / 19 / 80$ & -10.0 & 254 & ", \\
\hline-224 & $6 / 26 / 80$ & -9.0 & 170 & ", \\
\hline-225 & $6 / 30 / 80$ & -5.4 & 234 & ", \\
\hline-226 & $7 / 1 / 80$ & -19.6 & 1320 & $"$ \\
\hline-227 & $7 / 2 / 80$ & -20.2 & 560 & ", \\
\hline-228 & $3 / 31 / 82$ & -21.5 & - & Tennessee \\
\hline-229 & $3 / 31 / 82$ & -20.5 & 84 & $"$ \\
\hline-230 & $6 / 3 / 82$ & -14.8 & 181 & $"$ \\
\hline-231 & $1 / 8 / 80$ & -19.2 & - & Utah \\
\hline-232 & $1 / 31 / 80$ & - & 228 & " \\
\hline-233 & $2 / 20 / 80$ & -8.6 & 351 & "" \\
\hline-234 & $2 / 26 / 80$ & -8.6 & 135 & " \\
\hline-235 & $3 / 12 / 80$ & -9.4 & 220 & $"$ \\
\hline
\end{tabular}

